Casos C línicos

Arch. Esp. Urol., 58, 2 (164-167), 2005

\section{SCHWANNOMA ANTIGUO: VARIANTE RARA DE SCHWANNOMA.}

Ana M aría Grasa Arnal, Jorge Abad Roger, Araceli Bono Ariño, Jesús Javier Vera Álvarez ${ }^{1}$ y José Ignacio Sanz Vélez.

Servicio de Urología y Sección de Anatomía Patológica ${ }^{1}$. Hospital San Jorge. Huesca. España.

Resumen.- OBJETIVO: A propósito de un caso de schwannoma retroperitoneal con características histológicas poco frecuentes, se evalúa su significación clínica e indicaciones de manejo terapeutico.

MÉTODOS: Presentación de un caso de schwannoma retroperitoneal benigno, como hallazgo incidental, al practicar una Resonancia Magnética, en una paciente de 70 años cuya clínica era de una lumbociática de seis meses de evolución. Revisión bibliográfica.

RESULTADO S: Tras la exéresis quirúrgica se llegó al diagnóstico de schwannoma retroperitoneal antiguo. No se administra ningún tratamiento complementario. No se ha evidenciado recidiva durante seguimiento. Los cambios histológicos que se ven en los casos de schawannoma antiguo se han interpretado como degenerativos, indicando una larga evolución, que ha motivado su denominación de "antiguos". Algunos de los casos fueron diagnosticados como malignos.

Ana María Grasa Arnal

Avda. de Menéndez Pidal no 11, piso $20 \mathrm{C}$

22003 Huesca. España

e-mail: agrasa@telefonica.net

Trabajo recibido: 21 de junio 2004
CON CLUSIO N ES: El schwannoma antiguo es una variante poco habitual del schwannoma común benigno. M icroscópicamente muestra áreas de tejido hipocelular y núcleos pleomórficos sugestivos de malignidad. Su manejo terapéutico es similar al de otros procesos benginos retroperitoneales, mediante exéresis quirúrgica y seguimiento.

Palabras clave: Tumores retroperitoneo. Schwannoma benigno.

Summary.- O BJECTIVES: W e report one case of retroperitoneal schwannoma with unfrequent histological characteristics evaluating its clinical significance and therapeutic options.

METHODS: W e report the case of a benign retroperitoneal schwannoma as an incidental M RI finding in a 70 -yearold female patient with symptoms of lumbosciatic pain for 6 months. We perform a bibliographic review.

RESULTS: After surgical excision the diagnosis was retroperitoneal old schwannoma. No additional treatment was given. There is no evidence of recurrence on follow-up. The histological changes apparent in the cases of old schwannoma have been interpreted as degenerative, indicating a long evolution, which has motivated its denomination as old. Some cases have been diagnosed as malignant

CONCLUSIONS: The old schwannoma is a rare variety of the benign common schwannoma. $M$ icroscopically its shows areas of hypocellular tissue and pleomorphic nuclei suggestive of malignancy. Therapeutic management is similar to other benign retroperitoneal processes, surgical excision and followup.

Keywords: Retroperitoneal tumors. Benign schwannoma.

\section{INTRODUCCIÓN}

La mayor parte de los tumores que se desarrollan en retroperitoneo lo hacen a partir de estirpes celulares que embriológicamente derivan de estructuras contenidas en este espacio (neuroectodermo, mesodermo y restos embrionarios). Se trata de neoformaciones poco frecuentes. Su incidencia es baja $(0,3-3 \%$ del total de neoplasias). Ackerman los clasifica en dos grupos: los de 


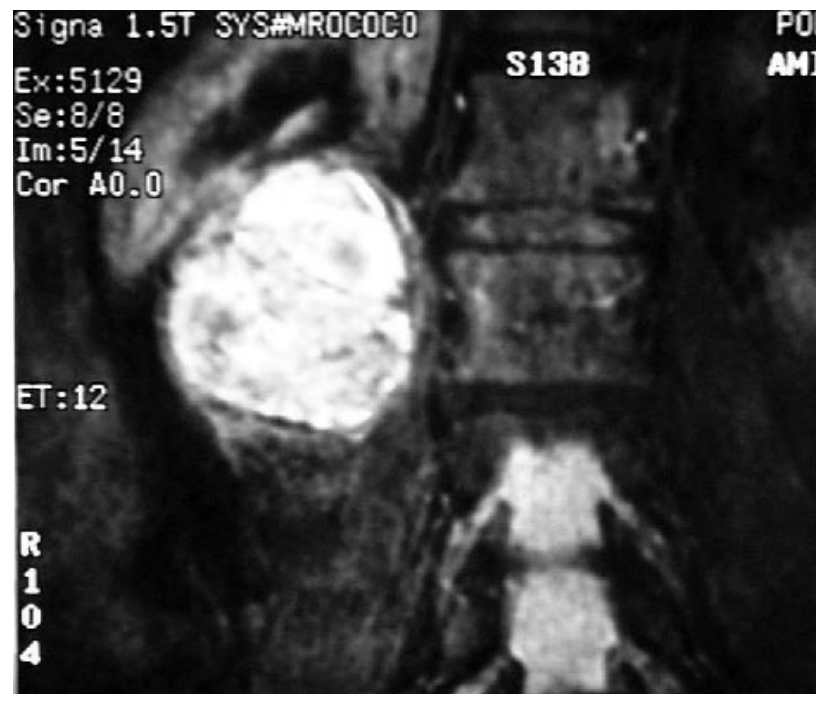

FIG URA 1. R.N.M. : Imagen redondeada de $6 \mathrm{~cm}$., encapsulada y heterogénea. Desde nivel de hilio renal derecho, desplazando cava y polo inferior renal.

origen nervioso y los de origen embrionario. A proximadamente el 60-85\% son malignos, de ellos, el $75 \%$ son mesodérmicos y el $25 \%$ de origen nervioso. El resto, son lesiones benignas.

\section{CASO CÚNICO}

Se trata de una mujer de 70 años, que tiene como antecedentes de interés: varicectomía en EEll; crisis hipertensivas; léntigos solares faciales; quistes seborreicos de pequeño tamaño en cuero cabelludo y tronco; osteoporosis y lumbalgias de repetición.

La paciente acudió a nuestra consulta remitida por hallazgo radiológico de una lesión ocupante de espacio en situación retroperitoneal paravertebral derecha (Figura 1). La RN M se había realizado por un cuadro de lumbociática con larga evolución.

En la anamnesis no refiere sintomatología miccional, ni hematuria ni cambios en el tránsito intestinal.

En la exploración física el abdomen es blando, depresible, sin masas, sin globo vesical y sin puntos dolorosos a nivel renoureteral e hipogastrio. No edemas en EEll.

Se realizó TAC abdominal con contraste (Figura 2), donde se confirmó una masa retroperitoneal, no afectando riñones ni órganos adyacentes.

Se decide intervención quirúrgica de la paciente en la

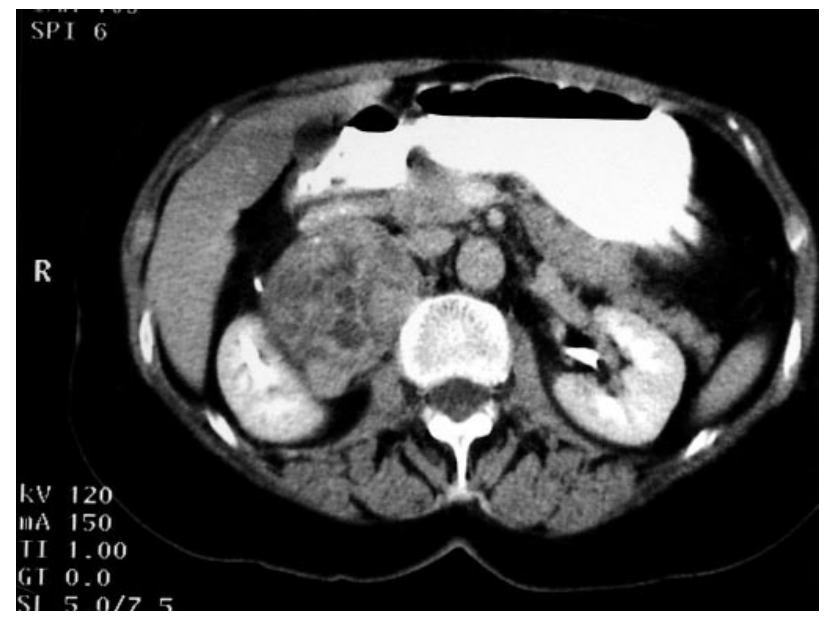

FIG URA 2. T.A.C. abdomen: Masa heterogénea con alguna calcificación, delimitada excepto en región distal de psoas. Riñones normales.

que se extirpa una pieza redondeada (Figura 3 ), que mide $7 \times 6,5 \times 4,3 \mathrm{~cm}$. y pesa 123,6 grs. El postoperatorio no presentó incidencias especiales.

A los 8 meses de la cirugía, la paciente no presenta signos de recidiva de su enfermedad. Mantiene su clínica previa de lumbociática.

\section{Diagnóstico anatomo-patológico:}

Se han realizado técnicas de inmunoperoxidasa habiéndose observado positividad para proteína S-100 y negatividad para la Desmina y la Actina de músculo liso. No se han evidenciado signos de malignidad.

Schwannoma con signos degenerativos (Schwannoma antiguo).

\section{DISCUSIÓN}

Los schwannomas derivan de las células de Schwann, que son células satélites del Sistema N ervioso Periférico. Son tumores muy poco frecuentes. Su distribución más habitual es en nervios periféricos de cuello, cabeza y extremidades.

Histológicamente, presentan zonas de alta celularidad (tejido Antoni A), y de baja celularidad, (tejido Antoni B). En el tejido Antoni A pueden estar presentes unas estructuras denominadas cuerpos de Verocay, acúmulos de núcleos en empalizada. Las áreas tipo A ntoni B están incluídas en una matriz laxa y edematosa con vasos sanguíneos prominentes, debido a un engrosamiento hialino 


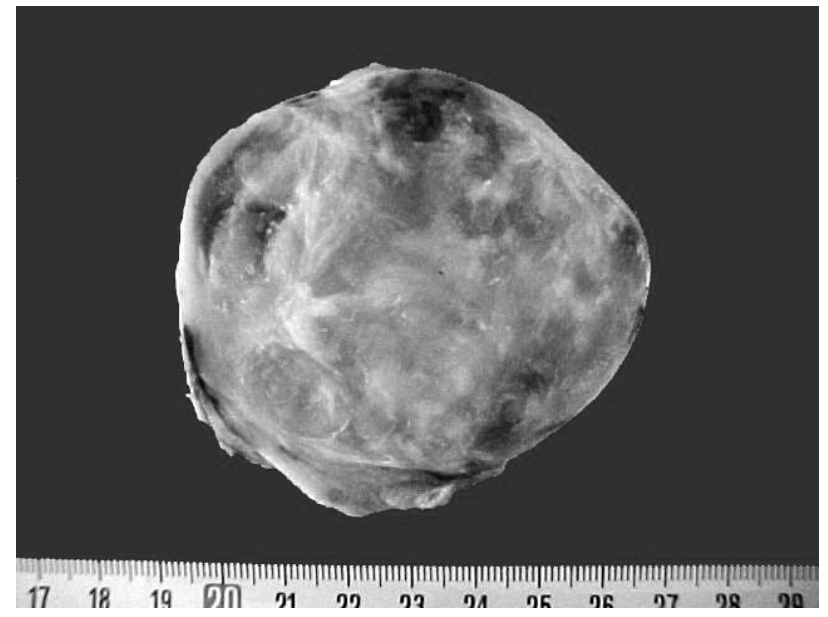

FIG URA 3. Pieza exeresis: Superficie externa pardo-grisácea. Consistencia blanda.

rodeado de los núcleos de las células tumorales, dispuestos en pseudoempalizada (4).

A parecen entre la 3a y la 7ạ décadas de la vida, sin tener predilección por ningún sexo. En las personas afectadas de enfermedad de Von Recklinghausen, la incidencia de tumores neuroectodérmicos es más elevada, el comportamiento es más agresivo y aparece en edades más tempranas. (4-6).

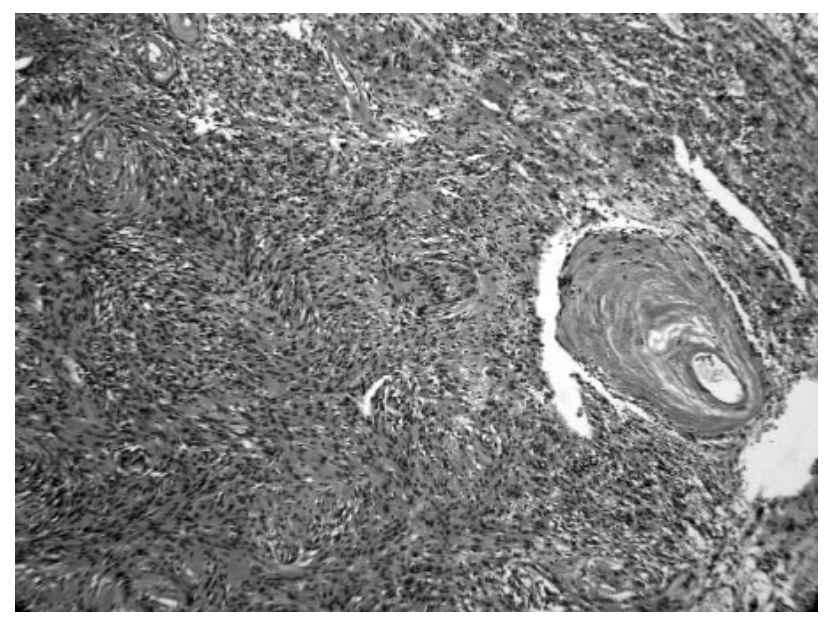

FIG URA 4. Examen microscópico: Patrón celular alternante, con áreas de aspecto laxo y edematoso, células a largadas, de ordenamiento irregular con zonas de degeneración quística y otras colagenizadas. 0 tras áreas marcadamente celulares, con núcleos alargados en empalizada, formando en ocasiones cuerpos de Verrocay.
La clínica es inespecífica. Los síntomas suelen estar relacionados con la compresión de estructuras vecinas por el gran crecimiento de la masa. (3).

El schwannoma antiguo es una variedad del schwannoma común benigno. Cuando se localiza en retroperitoneo suele ser un tumor de gran tamaño. Clínicamente no pueden diferenciarse, por lo que el tratamiento es idéntico para ambos, la exéresis quirúrgica.

El schwannoma antiguo es de baja agresividad. Se caracteriza por cambios degenerativos tales como grandes áreas de tejido hipocelular y otros hallazgos atípicos (hipercromatismo nuclear, núcleos gigantes, células binucleadas, células gigantes multinucleadas, etc.) que, en un principio, sugieren malignidad, pero sin embargo, no implican mal pronóstico. Puede aparecer una degeneración mixoide o xantomatosa (2). La degeneración epitelioide maligna de los schwannomas es un proceso muy infrecuente.

A natomopatológicamente tiene un gran parecido con otras neoplasias, algunas malignas. Es importante hacer el diagnóstico diferencial con los sarcomas de lento crecimiento, los schwannomas malignos y otras neoplasias malignas del tejido nervioso (2).

Los estudios iniciales de imagen (R.M.N., T.A.C., ecografía, etc.), deben completarse con el estudio anatomopatológico de la pieza, que confirmará el diagnóstico (3).

El tratamiento más adecuado es la extirpación quirúrgica completa de la masa, y aunque generalmente, el pronóstico es muy bueno en los casos benignos, debe seguirse muy de cerca al paciente, ya que se han descrito casos de malignización, sobre todo si se asocia a la enfermedad de Von Recklinghausen. También se describe alguna recidiva por extirpaciones incompletas de las lesiones (5).

\section{BIBUOGRAFIA y LECTURAS RECOMENDADAS (*lectura de interés y **lectura fundamental)}

**1. ACKERMAN, L.V.: "Tumors of the retroperitoneal mesenterio and peritoneum". Atlas of tumor pathology. Washington. DC: Armed forces institute of pathology, National Research Council 11, 23: 136, 1954.

*2. LEDESMA, C.; PORTILLA, J.; HERNÁNDEZ, F. y cols.: "Schwannoma antiguo paraglandular". Medicina Oral, 4: 398, 1999.

*3. DE DIEGO, E.; ROCA, A.; MARTÍN, B. y cols.: "Schwannoma benigno retroperitoneal. Aportación de un nuevo caso". Actas Urol. Esp., 24: 685, 2000. 
4. CONDE, J.M.; ESPINOSA, J.; CAMACHO, E. y cols.: "Schwannoma presacro benigno que provoca uropatía obstructiva. Caso clínico y revisión de la literatura". Actas Urol. Esp., 25: 237, 2001.

5. POZO, B.; PÉREZ, I.; FERNÁNDEZ, E. y cols.: "Schwannoma retroperitoneal benigno en paciente con antecedentes de tumor de Wilms en la infancia". Actas Urol. Esp., 27: 379, 2003.

6. VEGA, M.; ORLICH, C.; VALVERDE, H.: "Schwannoma de colon. Reporte de un caso". Acta Médica Costarricense 45, 2: 77, 2003.

7. ESCLARIN, M.A.; SANZ, J.I.; ORTAS J.L.. y cols.: "Tumor retroperitoneal infrecuente: schwannoma benigno". Actas Urol. Esp., 15: 577, 1991.

8. GIRONÉS, J.; ROIG, J.; RODRÍGUEZ, J.I. y cols.: "Schwannoma retroperitoneal maligno asociado a neurofibromatosis". Cirugía Española, volumen 73, $n^{\circ} 4$ : 255, 2003.

**9. BALIBREA, J.L.: "Afecciones quirúrgicas retroperitoneales". En Tratado de Cirugía. Editorial Marban S.L. Madrid. España, 1943, 1994.

*10. GALOFRÉ, N.; SUPERVÍA, A.; PEÑA, M.J. y cols.: "Schwannoma benigno retroperitoneal". An. Med. Int. Madrid, 13: 568, 1996.

Casos C línicos

Arch. Esp. Urol., 58, 2 (167-170), 2005

\section{GANGRENA DE FOURNIER SECUNDARIA A CATETERISMO URETRAL}

José Carlos López Pacios², José M aría Sánchez $M_{\text {erino }}{ }^{1}, M$. C armen Piñeiro Fernández ${ }^{2}, M$. Bouso M ontero ${ }^{3}$, Luis Parra M untaner ${ }^{1}$ y Jesús $G$ arcía Alonso ${ }^{1}$.

Servicios de Urología ${ }^{1}$ Urgencias $s^{2}$ Ana tomía Patológica ${ }^{3}$ del Hospital del Bierzo. FuentesnuevasPonferrada. León. España.

José Carlos López Pacios

C/ Ancha $1450 \mathrm{~A}$

24400 PO N FERRADA.

León. España

Trabajo recibido: 30 de junio 2004
Resumen.- O BJETIVO S: M ediante el forma to de caso clínico presentamos un caso de $G$ angrena de Fournier secundario a cateterismo uretral.

M ÉTO DO S: Realizamos una descripción del caso clínico que nos ocupa, en el que se ha identificado la causa inicial, así como una breve revisión bibliográfica de esta patología.

RESULTADO : Aunque la gangrena de Fournier se consideraba inicialmente como de etiología idiopática, en la actualidad es posible identificar en la mayoría de los casos la puerta de entrada de la infección. En nuestro caso la colocación de un catéter uretral fue el mecanismo de inicio de la infección, asociado a dos factores como la diabetes y el alcoholismo que favorecen el desarrollo de la misma. Se trató mediante desbridamiento quirúrgico y se realizó uretrectomía parcial. El paciente fue finalmente exitus.

CON CLUSIO NES: Se debe llamar la atención sobre la necesidad de que la manipulación uretral sea realizada por manos expertas dada la gravedad de las posibles complicaciones.

Hay que insistir en la necesidad de un tratamiento precoz con una cobertura de antibióticos de amplio espectro, un desbridamiento radical y una revisión urológica completa.

Palabras clave: G angrena de Fournier. C ateterismo. Uretra.

Summary.- O BJECTIVES: W e report one case of Fournier's gangrene secondary to urethral catheterization. METHODS: W e describe the clinical case, in which the initial cause was identified, and perform a short bibliographic review.

RESULTS: Although Fournier's gangrene was initially considered as idiopathic in etiology, currently it is possible to identify the entrance site of the infection. In the present case the insertion of a urethral catheter was the starting mechanism, associated with factors such as diabetes and alcoholism which favour its development The patient was treated by surgical debridement and partial urethrectomy but finally died.

CON CLUSIO N S: W e want to point out that urethral instrumentation should be done by expert hands due to the severity of possible complications. We should insist in the need of precocious treatment with wide spectrum antibiotics, radical debridement and complete urologic evaluation. 